\section{Prolegómenos}

Derecho y Valores
Prolegómenos. Derechos y Valores

ISSN: 0121-182X

derechos.valores@umng.edu.co

Universidad Militar Nueva Granada

Colombia

Mora Bastidas, Freddy Alberto

\title{
VALORACIÓN DEL SERVICIO PÚBLICO DE SEGURIDAD SOCIAL VENEZOLANO: A 5 AÑOS DE
}

\section{SU CREACIÓN}

Prolegómenos. Derechos y Valores, vol. XI, núm. 22, julio-diciembre, 2008, pp. 171-180

Universidad Militar Nueva Granada

Bogotá, Colombia

Disponible en: http://www.redalyc.org/articulo.oa?id=87602211

Cómo citar el artículo

Número completo

- Más información del artículo

Página de la revista en redalyc.org

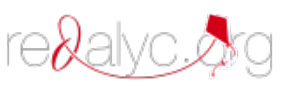

Sistema de Información Científica

Red de Revistas Científicas de América Latina, el Caribe, España y Portugal Proyecto académico sin fines de lucro, desarrollado bajo la iniciativa de acceso abierto 
Prolegómenos - Derechos y Valores

\section{VALORACIÓN DEL SERVICIO PÚBLICO DE SEGURIDAD SOCIAL VENEZOLANO: A 5 AÑOS DE SU CREACIÓN*}

\author{
M. Sc. Freddy Alberto Mora Bastidas**
}

\section{Resumen}

Con la presente investigación se valoró el servicio de seguridad social venezolano a 5 años de su creación. Se puede afirmar que el constituyente de 1999 creo el Servicio Publico de Seguridad Social y garantizo el derecho a la seguridad social, convirtiéndose en una política de Estado destinada a la atención de los habitantes en un conjunto de áreas o contingencias familiares, personales y sociales que inciden en las relaciones laborales. En la presente investigación documental de tipo descriptiva y analítica se concluye que luego de cinco (5) años de la creación legal del Sistema de Seguridad Social, el Estado no ha logrado la implementación del Servicio Publico de Seguridad Social, afectando no solo el cumplimiento del mandato contenido en el Art. 2, 3 y 86 constitucional; desatendiendo la protección social de todos los beneficios del Sistema. Con la Creación del Servicio Publico de Seguridad Social se debe complementar el régimen legal de protección social de las relaciones laborales, pero contradictoriamente existe una omisión y retardo por parte del Estado en el aseguramiento del derecho a la seguridad social debido al retardo en la aprobación de dos (2) leyes de desarrollo y la negativa del Estado en la activación de las funciones de la Tesorería y la Superintenden-

\footnotetext{
* Estos comentarios constituyen un resultado del proyecto de investigación N. E-286 -08-09-B, titulado "El acceso a la justicia y la tutela judicial en materia contencioso administrativo laboral y contencioso de la seguridad social", financiado por el CDCHT - ULA. La versión preliminar de este artículo fue enviado al VI congreso regional de las américas 2008, siendo aceptado y el abstract fue publicado en las memorias del congreso.

** Abogado litigante. Magíster en Derecho Laboral. Especialista en Derecho Administrativo. Profesor Ordinario (Programa de Promoción al Investigador (PPI) NIVEL I). (Universidad de Los Andes - Mérida - Venezuela). fremoba@ula.ve http://webdelprofesor.ula.ve/economia/fremoba.
}

cia de la Seguridad Social. Se sugiere cumplir en un lapso perentorio con el desarrollo legal y reglamentario del Sistema de Seguridad Social y por un lado la implementación inmediata del Sistema de Seguridad Social para lograr el desarrollo de un Estado de justicia social.

\section{Palabras clave:}

Sistema de Seguridad Social, derecho a la seguridad social, estado de bienestar, políticas públicas, relaciones laborales, sistemas prestacionales, regímenes prestacionales.

\section{ASSESSMENT OF PUBLIC SERVICE OF VENEZUELAN SOCIAL SECURITY: 5 YEARS OF ITS CREATION}

\begin{abstract}
With this investigation we have evaluated the social security service Venezuelan 5 years of its creation. It can be said that the Constitution of 1999 created the Public Service and Social Security guarantees the right to social security, becoming a state policy to the attention of people on a set of contingency areas or family, personal and social impact on labor relations. In this documentary research of descriptive and analytical concludes that after five (5) years of the legal establishment of the Social Security System, the State has failed to implement the public social security system, affecting not only the implementation of mandate contained in Article 2, 3 and 86 constitutional neglecting the welfare of all the benefits of the system. With

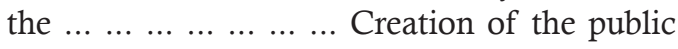
social security system should complement the legal system of social protection of labor relations, but there is a failure and contradictorily delay by the State in securing the right to social security due to the delay in the approval of two (2) development laws and the refusal of the state in the activation of the functions of the Treasury and the Superintendency of Social Security. It is suggested to meet a mandatory period in the development of legal and regulatory social security system on the one hand and the immediate implementation of the Social
\end{abstract}


Security System to ensure the development of a state of social justice.

\section{Key words}

Social Security System, the right to social security, welfare state, public policy, industrial relations systems, benefits, benefits schemes.

\section{PLANTEAMIENTO DE LA INVESTIGACIÓN}

En el título V de la Ley Orgánica del Sistema de Seguridad Social se establece en los artículos 117 y $118^{1}$ el lapso para la nueva institucionalidad y el periodo de implantación - que el autor considera debe llamarse "periodo de implementación del Sistema" -. Estos dos artículos tienen una concatenación, pues el artículo 117 impone una obligación al Ejecutivo Nacional para desarrollar un plan que permita la implantación de la nueva institucionalidad del Sistema de Seguridad Social y el artículo 118 establece un lapso de cinco (5) años, como período para la implementación del Sistema de Seguridad Social.

El treinta (30) de diciembre de dos mil siete (2007), se cumplieron los cinco (5) años del período de implementación del Sistema de Seguridad Social y hasta los momentos continuamos con "la transitoriedad permanente de la Seguridad Social" y solamente existen avances en materia de condiciones y medio ambiente del trabajo.

Estos comentarios constituyen un avance del proyecto de investigación $\mathrm{N}^{\circ} \mathrm{E}-286-08-09-\mathrm{B}$, intitulado "El acceso a la justicia y la tutela judicial en materia contencioso administrativo laboral y

La Ley Orgánica del Sistema de Seguridad Social, fue reformada en el paquete de leyes que conforman la habilitante (2008), aprobada bajo el Decreto $\mathrm{N}^{\mathrm{o}} 6243$ y publicada en Gaceta Oficial No 5891. En la mencionada reforma, solamente fueron modificados parcialmente los siguientes artículos: 28, 43, 51, 104, 112, 113 y 116. contencioso de la seguridad social", financiado por el CDCHT - ULA. La versión preliminar de este artículo fue enviado al VI Congreso Regional de Las Américas 2008, siendo aceptado y el abstract fue publicado en las memorias del Congreso.

Luego de cinco (5) años, durante los cuales el Estado tenía la obligación constitucional y legal de la implementación del Sistema de Seguridad Social, es idóneo citar una apreciación de Díaz (2003) sobre el Sistema de Seguridad Social, en la cual indica que la Constitución de 1999 establece los principios, las ramas asegurativas y el sentido progresivo de la disciplina, pero que no están las coordenadas lo suficientemente claras. Afirma que el Estado tiene un comportamiento o desempeño con menos respuestas ${ }^{2}$. Con esta valoración del período de implementación del Sistema de Seguridad Social, se darán algunos aportes que permitirán a los distintos actores sociales, lograr la perfección del Servicio Público de Seguridad Social para asegurar el derecho a la Seguridad Social de todos los beneficiarios del Sistema.

Debido a la relevancia y actualidad del tema: "Servicio Público de Seguridad Social", se busca descubrir: ¿Cuál ha sido el desarrollo normativo en el período de implementación del Servicio Público de Seguridad Social?; y ¿Cuáles son las medidas que pueden permitir el desarrollo de la actividad administrativa del Servicio Público de Seguridad Social?

\section{OBJETIVOS DE LA INVESTIGACIÓN}

\section{Objetivo general}

Examinar el Servicio Público de Seguridad Social en el período legal de implementación del Sistema.

2 Díaz, Luis Eduardo. ¿Acumulación de pobreza o reparto de riqueza?: El dilema de la seguridad social en Venezuela. Revista de Ciencias Sociales, mayo 2003, vol. IX, no.2, p.215-234. ISSN 1315-9518. 
Objetivos específicos

1) Estudiar la articulación constitucional del Servicio Público de Seguridad Social.

2) Revisar la estructura organizativa del Sistema de Seguridad Social.

3) Analizar algunos aspectos de los procedimientos administrativos y contencioso administrativo de la Seguridad Social.

\section{METODOLOGÍA}

La investigación a desarrollar es de la modalidad jurídico - dogmática, de carácter documental que permitirá al investigador examinar el período de implementación del Servicio Público de Seguridad Social a 5 años de su creación con el propósito de ampliarlo y profundizarlo con el apoyo de fuentes bibliográficas y documentales.

De acuerdo con el planteamiento del problema y los respectivos objetivos de la investigación, el diseño de la misma se ubica dentro de una investigación documental, debido a que se pretende recopilar, analizar y seleccionar toda la información plasmada en libros, leyes, y los demás documentos relacionados con el tema, con la finalidad de llegar a un conocimiento y comprensión profunda.

\section{ARTICULACIÓN DEL DESARROLLO CONSTITUCIONAL DEL SERVICIO PÚBLICO DE SEGURIDAD SOCIAL}

El artículo 86 constitucional consagra el derecho a la seguridad social y la creación del Servicio Público de Seguridad Social - que el constituyente lo denomina Sistema de Seguridad Social - e igualmente consagra los principios generales que servirán de soporte para el desarrollo legislativo de la normativa necesaria a los fines de la implementación del Servicio Público de Seguridad Social.

El primer grupo de normas que deben articularse para el óptimo funcionamiento del Servicio Público de Seguridad Social son las siguientes: Artículo 2, 3 y 4 constitucional. Tanto el Poder Legislativo como el Poder Ejecutivo, al momen- to de dictar las leyes y Reglamentos que permitan el desarrollo del Sistema de Seguridad Social tienen que estar claros sobre la concepción del Estado, los valores constitucionales y los fines del Estado, en donde las distintas actividades del Servicio Público de Seguridad Social, estarán destinadas a la procura de la justicia social.

El segundo grupo de normas, permiten el desarrollo del Servicio Público de Seguridad Social y se encuentra conformada por los artículos $136,156,137,139,140,141,19,21,23,25,51$, $62,75,76,78,79,80,81,82,87,88,89,91$, $92,100,111,122,123$ y 147 constitucional. A su vez estas normas constitucionales se pueden sub - agrupar de la siguiente forma: Un conjunto de normas que tienen incidencia sobre las actividades del Servicio Público de Seguridad Social; otras normas que servirán para el desarrollo del Sistema de Seguridad Social.

En relación con las normas que tienen incidencia sobre las actividades del Servicio Público de Seguridad Social nos encontramos con el principio constitucional de colaboración entre los distintos órganos del Poder Público, (artículo 136 constitucional), por medio del cual los órganos que conforman la distribución vertical y horizontal del Poder Público tienen la obligación de colaborar para el cumplimiento de los fines del Estado y la materia de Seguridad Social no escapa de ese mandato constitucional, toda vez que es necesaria la participación de los distintos Poderes Públicos Nacionales para el cumplimiento de los objetivos del Sistema de Seguridad Social (Formación de leyes, Elaboración de Reglamentos, Diseño de manuales de organización y funcionamiento, Diseño de planificación) y en algunas circunstancias la participación del Poder Estadal y Municipal (Participación y control social). El artículo 156 constitucional, establece la competencia del Poder Público Nacional, en donde se reserva en materia legal, el desarrollo de las leyes del trabajo y de la seguridad social.

El principio de la legalidad contenido en el artículo 137 constitucional, permite encuadrar la actividad administrativa del Servicio Público 
de Seguridad Social al mandato constitucional y legal, generando supuestos de responsabilidad individual (artículo 139), responsabilidad patrimonial del Estado (artículo 140) y contemplando la posibilidad de responder civil, penal y administrativamente (artículo 25).

El principio constitucional para la organización de la Administración Pública, contemplado en el artículo 141, es determinante para el éxito de la implementación del Servicio Público de Seguridad Social, toda vez, que en los distintas actividades de los Regimenes Prestacionales se deben establecer reglas y parámetros claros, expeditos, eficaces y eficientes, que permitan el cumplimiento de los objetivos del Sistema de Seguridad Social; en otras palabras, se deben regular los distintos procedimientos administrativos y mecanismos para la reclamación, denuncias y quejas por el funcionamiento anormal o deficiente del Servicio Público de Seguridad Social. Igualmente dentro de la organización administrativa se puede incluir el principio contenido en el artículo 51 constitucional, referido al derecho que tiene todo ciudadano a dirigir solicitudes o peticiones a los órganos del Poder Público, necesario en las prestaciones que debe suministrar el Servicio de Seguridad Social, porque en muchos casos se activan a instancia de parte.

Dentro de la nueva concepción del Estado Venezolano, la participación ciudadana - (artículo 62 constitucional) - es indispensable en la formación, ejecución y control de la gestión pública. La participación de los actores sociales en la ejecución de actividades del Servicio Público de Seguridad Social se encuentra consagrada en la Ley Orgánica del Sistema de Seguridad Social como un mecanismo de control social ${ }^{3}$.

En relación con las normas que sirven para el desarrollo del Sistema de Seguridad Social se

El artículo 23 de la Ley Orgánica del Sistema de Seguridad Social indica lo siguiente: "Corresponde al Ejecutivo Nacional la creación de los órganos de consulta, seguimiento $y$ control para la participación ciudadana en las instituciones del Sistema de Seguridad Social. Estos órganos deberán estar integrados por los actores sociales vinculados a la seguridad social y por otros, cuya participación contribuya a hacer encuentran las normas que protegen los derechos sociales y de las familias. Los artículos 75 y 76 constitucional imponen al Estado la protección de la familia, la maternidad y la paternidad. Esta protección se encuentra conectada con las contingencias que garantizan el derecho a la seguridad social ${ }^{4}$. Los artículos 78 y 79 constitucional garantizan la protección integral de los niños, niñas y adolescentes, consagrando su incorporación al Servicio Público de la Seguridad Social en las disposiciones de la Ley Orgánica de Protección al Niño y al Adolescente.

El Estado por mandato del artículo 80 y 81 de la Constitución de la República Bolivariana de Venezuela, tiene la obligación de proteger a los ancianos y las personas con discapacidad, formando parte estas obligaciones de las contingencias contenidas en el artículo 86 constitucional, que han sido desarrolladas por el Régimen Prestacional de Previsión Social.

El derecho a la vivienda - contemplada en el artículo 82 constitucional - ha sido desarrollado ampliamente por el legislador en la Ley del Régimen Prestacional de Vivienda y Hábitat ${ }^{5}$.

efectivo el derecho de las personas a la seguridad social". En la Ley de Servicios Sociales se regula los mecanismos de consulta, participación ciudadana y control, con la creación del Consejo Nacional de Servicios Sociales - artículos 84 al 86 - , crea la figura de los Comités Comunitarios de Servicios Sociales - artículo 89 y 90 - y las Asambleas de los Comités Comunitarios. En la Ley del Régimen Prestacional de Empleo, la participación ciudadana y el control social se encuentran contenidos en el Titulo VII, Capítulo I, en donde se establecen las reglas para el funcionamiento de las Asambleas y Comités Comunitarios de Activación Socioproductiva. En el caso de la Ley Orgánica de Prevención, Condiciones y Medio Ambiente del Trabajo, en el Título III, Capítulo I, se crea la figura del delegado de prevención y del Comité de Seguridad y Salud Laboral.

4 En el ordenamiento laboral y de la seguridad social existe el Título VI "De la Protección Laboral de la Maternidad y la Familia", incluido dentro de la Ley Orgánica del Trabajo y la novedosa Ley de Protección a la $\mathrm{Pa}$ ternidad y la Familia, publicada en Gaceta Oficial $N^{\circ}$ 38.773 de fecha veinte (20) de septiembre de 2007.

5 La Ley del Régimen Prestacional de Vivienda y Hábitat, fue reformada en el paquete de leyes que conforman la habilitante (2008), aprobada bajo el Decreto $\mathrm{N}^{\circ}$ 6072 y publicada en Gaceta Oficial No 5889. 
La protección a la Salud - consagrada desde el artículo 83 al 86 del texto constitucional - ha sido una de las contingencias del Sistema de Seguridad Social que hasta los momentos no han sido desarrolladas legalmente por parte del Poder Legislativo Nacional, existiendo un retraso que supera el período de implementación del Sistema de Seguridad Social.

Los principios fundamentales que sustentan el desarrollo de la normativa legal del derecho individual del trabajo - artículos 87 al 92 - deben articularse con el conjunto de normas del Sistema de Seguridad Social, a los fines de la protección integral de los trabajadores dependientes y de los trabajadores no dependientes.

El derecho a la recreación consagrada en el artículo 111 de la Constitución de la República Bolivariana de Venezuela, forma parte de las contingencias del derecho a la seguridad social contenidas en el artículo 86 constitucional, quedando a cargo del Régimen Prestacional de Previsión Social, específicamente bajo las directrices del Instituto Nacional de Capacitación y Recreación de los Trabajadores.

En los artículos 100, 122, 123, 147 y 328 se regula la protección de los trabajadores de la cultura, de los pueblos indígenas, de los funcionarios públicos y de los miembros de las Fuerzas Armadas, que se encuentran garantizados por el principio constitucional de universalidad en relación al disfrute del derecho a la Seguridad Social.

El tercer grupo de normas, permiten la planificación, así como el control administrativo, fiscal y jurisdiccional del Servicio Público de Seguridad Social y se encuentra conformada por los artículos 259, 262, 274, 281, 289, 299, 310, $311,313,334$ y 335 constitucional.

\section{ESTRUCTURA ORGANIZATIVA (ENTES Y ORGANOS DE LAS LEYES DE DESARROLLO)}

El Título II de la Ley Orgánica del Sistema de Seguridad Social, desarrolla los parámetros de la estructura organizativa y funcional del Sistema de Seguridad Social; sin embargo, cada uno de los Sistemas Prestacionales, se encuentran incorporados dentro de la estructura organizativa y funcional del Sistema de Seguridad Social.

\subsection{Estructura organizativa y funcional del Sistema Prestacional de Salud}

Han transcurrido más de cinco (5) años, sin que el Poder Legislativo dictare la Ley Orgánica del Sistema Prestacional de Salud y solamente en la actualidad el artículo 54 de la Ley Orgánica del Sistema de Seguridad Social contempla la integración de todas las estructuras, órganos, programas y servicios que sostengan parcial o totalmente la política de salud.

\subsection{Estructura organizativa y funcional del Sistema Prestacional de Previsión Social}

\subsubsection{Régimen Prestacional de Empleo}

La estructura organizativa y funcional del Regímen Prestacional de Empleo se encuentra conformada por: 1) La Rectoría representada por el Ministerio con competencia en materia de empleo; 2) Los entes y órganos de gestión, representados por el Instituto Nacional de Empleo, con personalidad jurídica y patrimonio propio que se encuentra integrado por un Directorio, encargado de la atención integral de la fuerza de trabajo en situación de desempleo y otorgará las prestaciones económicas y el Servicio de Migraciones Laborales, encargado de canalizar las solicitudes de requerimientos de trabajadores y trabajadoras extranjeros con el objeto de autorizar su ingreso al mercado de trabajo; 3 ) Instancias de consulta y participación, representado por el Sistema Nacional de Protección Frente a la Pérdida del Empleo y el Desempleo, que a su vez, se encuentra integrado por la Red de Servicios de Atención Integral a la Persona en Situación de Desempleo, conformada por las instancias y organizaciones gubernamentales, sociales y comunitarias que desarrollan una gestión de servicios públicos, asociadas para dar respuesta integral a la persona en situación de 
desempleo, desarrollando las siguientes actividades: a) La promoción de programas de capacitación; b) Asesoría para la reconversión laboral; c) Información laboral sobre oportunidades de empleo, capacitación y ocupación socioproductiva; d) Información y orientación sobre los servicios que ofrecen los diversos institutos de los regímenes prestacionales del Sistema de Seguridad Social. La segunda instancia es la Red de Observatorios Laborales, (a nivel nacional, estadal y municipal), creados por el Instituto Nacional de Empleo, encargados de conocer y analizar el comportamiento y situación del empleo a nivel nacional, para coordinar en el diseño de las políticas, programas y servicios de atención a la población. La tercera instancia son los Comités Comunitarios de Activación Socioproductiva, para la organización social y respuesta colectiva en función del ejercicio efectivo de los derechos regulados en el Régimen Prestacional de Empleo. Los Comités se constituyen en Asambleas de Comités Comunitarios Parroquiales, Estadales y Municipales.

\subsubsection{Régimen de Seguridad y Salud en el Trabajo}

La estructura organizativa y funcional del Régimen de Seguridad y Salud en el Trabajo se encuentra conformada por: 1) La Rectoría representada por el Ministerio con competencia en materia de seguridad y salud en el trabajo; 2) Los entes de gestión, representados por el Instituto Nacional de Prevención, Salud y Seguridad Laborales, con personalidad jurídica y patrimonio propio que se encuentra integrado por un Directorio, tiene como finalidad garantizar la promoción del trabajo seguro y saludable; el control de las condiciones y medio ambiente de trabajo, la prevención de los accidentes de trabajo y enfermedades ocupacionales, las prestaciones dinerarias y no dinerarias, políticas, programas, servicios de intermediación, asesorías, información y orientación laboral y por el Instituto Nacional de Capacitación y Recreación de los Trabajadores que tiene como finalidad garantizar a la población amparado por el Régimen la gestión directa de su infraestructura y programas, y la asociación o intermediación con servicios turísticos - recreativos del sector público, privado o mixto; 3) Órganos de supervisión, teniendo competencia las Unidades Técnico - Administrativas del Instituto Nacional de Prevención, Salud y Seguridad Laborales y las Unidades Técnico - Administrativas adscritas a la Inspectoría del Trabajo; 4) Organismos e instancia de consulta y participación, representado por el Consejo de Seguridad y Salud en el Trabajo, siendo la instancia asesora del órgano rector en las materias de promoción de la seguridad y salud en el trabajo, la prevención de los accidentes del trabajo y enfermedades ocupacionales, el control de las condiciones y medio ambiente del trabajo y la recreación, así como la utilización del tiempo libre, descanso y turismo social. La segunda instancia es el Comité de Seguridad y Salud Laboral conformado por el (los) delegado (s) o delegada (s) de prevención y por el empleador o sus representantes, teniendo la obligación de inscribirse por ante el Instituto Nacional de Prevención, Salud y Seguridad Laborales, teniendo como atribución la participación en la elaboración, aprobación, puesta en práctica y evaluación del programa de Seguridad y Salud en el Trabajo y promover las iniciativas sobre métodos y procedimientos para el control efectivo de las condiciones peligrosas de trabajo, proponiendo la mejora de los controles existentes o la corrección de las deficiencias detectadas. La tercera instancia son los Consejos Estadales, Municipales y Sectoriales integrados por los representantes de los empleadores ante los Comités de Seguridad y Salud Laboral; representantes de las organizaciones empresariales; representantes de las comunidades organizadas de la localidad con interés en el área de seguridad y salud en el trabajo; representantes de los institutos de educación superior con carreras en el área de seguridad y salud en el trabajo, recreación y utilización del tiempo libre; representantes de los centros de investigación en el área de salud y seguridad en el trabajo y la recreación; representantes de las organizaciones gremiales del área de seguridad y salud en el trabajo. 


\subsubsection{Régimen Prestacional de Servicios Sociales al Adulto Mayor y Otra Categorías de Personas}

La estructura organizativa y funcional del Régimen de Servicios Sociales al Adulto Mayory Otra Categoría de Personas se encuentra conformada por: 1) La Rectoría representada por el Ministerio con competencia en materia de servicios sociales al adulto mayor; 2) El ente de gestión, representados por el Instituto Nacional de Servicios Sociales, que tiene las siguientes competencias para realizar las actividades y gestiones necesarias en materia de protección, asistencia, atención, otorgamiento de asignaciones económicas a favor del adulto mayor y otra categoría de personas; 3) Órganos de gestión, teniendo competencia el Instituto Nacional de Servicios Sociales para crear a nivel estadal y municipal centros de servicios sociales que actuaran bajo la coordinación del Instituto Nacional de Servicios Sociales; 4) Organismos e instancia de consulta y participación, representado por el Consejo Nacional de Servicios Sociales siendo la instancia asesora del órgano rector en materia de servicios sociales. La segunda instancia son los Comités Comunitarios de Servicios Sociales, para la organización social y respuesta colectiva en función del ejercicio efectivo de los derechos regulados en el Regímen Prestacional de Servicios Sociales. Los Comités se constituyen en Asambleas de Comités Comunitarios Parroquiales, Estadales y Municipales.

\subsubsection{Régimen Prestacional de Pensiones $y$ otras asignaciones económicas}

Hasta los momentos el Poder Legislativo no ha dictado la Ley de Régimen Prestacional de Pensiones y Otras Asignaciones Económicas y a juicio del autor la estructura organizativa y funcional del régimen debería tener cierta similitud con los demás regimenes que conforman el Sistema Prestacional de Previsión Social.

\subsubsection{Estructura organizativa y funcional del Sistema Prestacional de Vivienda}

El Título II de la Ley del Régimen Prestacional de Vivienda y Hábitat establece las reglas que regulan el Sistema Nacional de Vivienda y Hábitat, conformado de la siguiente forma: 1) La Rectoría representada por el Ministerio con competencia en materia de vivienda y hábitat. Dentro de las disposiciones que regulan el Sistema Nacional de Vivienda, se encuentra el Capítulo II, destinado a regular la organización y funcionamiento del Banco Nacional de Vivienda y Hábitat, bajo la figura de Instituto Autónomo, adscrito al Ministerio con competencia en materia de vivienda y hábitat; 2) Los organismos de gestión, representados por el organismo integral estadal y municipal de vivienda y hábitat, el cual debe ser creado de conformidad con las reglas previstas en la Ley Orgánica de la Administración Pública. Igualmente se regula la promoción de los Consejos Parroquiales de Vivienda y Hábitat; 3) Instancias de participación del sector privado, representados por las empresas constructoras, los institutos de investigación y formación profesional y las formas asociativas comunitarias; así como por las operadoras financieras (instituciones financieras debidamente registradas y calificadas por el Banco Nacional de Vivienda y Hábitat y cooperativas de ahorro y crédito en vivienda y hábitat, calificada por el Banco $\mathrm{Na}$ cional de Vivienda y Hábitat); 4) Instancias de participación de los usuarios, a través de las organizaciones comunitarias integrales de vivienda y hábitat, encargados de la articulación de todas las asociaciones vecinales y sectoriales con la finalidad de acceder a los beneficios del Régimen Prestacional. Igualmente se contempla la figura de los Consejos Comunitarios de Vivienda y Hábitat que trabajaran de manera coordinada y complementaria con los Consejos Parroquiales de Vivienda y Hábitat.

Como se puede observar la estructura organizativa y funcional del Servicio Público de Seguridad Social es bastante compleja, debido a que se diseña con la participación de entes y órganos públicos, instancias de participación a nivel público y privado y la participación ciudadana, siendo imprescindible la aplicación de una eficiente planificación a los fines del cumplimiento de los objetivos del Sistema de Seguridad Social. Igualmente es necesario establecer 
parámetros para un idóneo reclutamiento y selección del personal que se encargará de operar el Servicio Público de Seguridad Social.

Méndez ${ }^{6}$, al estudiar la estructura organizativa y funcional del Sistema de Seguridad Social considera a la descentralización, como modelo típico de organización administrativa, atenuada con esquemas de coordinación e intersectorialidad, con lo cual el Sistema de Seguridad Social se enmarcara dentro de las nuevas tendencias de la organización del Poder Público en Venezuela.

El autor comparte parcialmente los comentarios de Méndez, pues desde el punto de vista jurídico la estructura organizativa y funcional del Servicio Público de Seguridad Social no se encuentra descentralizada, sino centralizada, ya que en todos los Regimenes la rectoría está a cargo del Ministerio (Poder Público Nacional) que a su vez depende de la Rectoría del Sistema (bajo el mando del Presidente de la República). Ahora, desde el punto de vista de la participación ciudadana (control en la gestión pública) y los criterios de desconcentración, el legislador hizo un esfuerzo por materializar estos principios en la organización del Servicio Público, dependiendo el éxito del sistema de la interrelación de los distintos componentes de la estructura organizativa y funcional con criterios de eficiencia, eficacia, responsabilidad, transparencia, responsabilidad, rendición de cuenta y sometimiento al principio de legalidad.

6 Méndez, A., "Estructura Organizativa y Funcional del Sistema de Seguridad Social", en Cuadernos de Postgrado N. 18, Comisión de Estudios de Postgrado, Universidad Central de Venezuela, Publicaciones UCV, Caracas 2004.

\section{DESARROLLO DE LOS PROCEDIMIENTOS ADMINISTRATIVOS (LEY RÉGIMEN EMPLEO ${ }^{7}$ Y LOPCYMAT)}

El artículo 1 de la Ley Orgánica del Sistema de Seguridad Social (LOSSS), establece el objeto de la Seguridad Social y de lo dispuesto en los artículos 52, 59, 64, 81, 96, 97 y 100 de la LOSSS se puede inferir la gran variedad de prestaciones que debe suministrar el Sistema de Seguridad Social, que pueden resultar en el otorgamiento de prestaciones económicas o de servicio por parte del Estado e igualmente por vía excepcional prestaciones económicas y de servicios impuestas por los Sistemas Prestacionales que deben ser satisfechas directamente por el patrono.

En la Ley del Régimen Prestacional de Empleo no se indican ni definen los distintos procedimientos administrativos laborales, sin embargo del análisis del articulado de la mencionada ley, - artículo 29 al 49 - se puede inferir la existencia de actividades que deben ser producidas por intermedio de los procedimientos administrativos no sancionatorios, y de los artículos 55 al 66, se puede inferir la existencia de actividades que deben ser producidas por intermedio de los procedimientos administrativos sancionatorios.

La Ley Orgánica de Prevención, Condiciones y Medio Ambiente del Trabajo, desarrolla el conjunto de normas y principios que regulan el funcionamiento del Régimen Prestacional de Seguridad y Salud en el Trabajo; sin embargo en su articulado no existe ninguna disposición relativa a los procedimientos administrativos.

En la Ley Orgánica de Prevención, Condiciones y Medio Ambiente del Trabajo, al igual que

\footnotetext{
El análisis del procedimiento administrativo en materia de Seguridad Social contenido en la Ley del Régimen Prestacional de Empleo, fue presentado en la III Jornadas de Investigación del Centro de Investigaciones y Desarrollo Empresarial de la Universidad de Los Andes (CIDE - ULA) y ha sido publicado en la revista arbitrada Visión Gerencial, Año 6. N Extraordinario de 2007.
} 
en la Ley del Régimen Prestacional de Empleo no se indican ni definen los distintos procedimientos administrativos laborales, por lo que es indispensable la aplicabilidad de la Ley Orgánica de Procedimientos Administrativos para regular el desarrollo de las actividades administrativas del Sistema.

\section{JURISDICCIÓN CONTENCIOSO ADMINISTRATIVA DE LA SEGURIDAD SOCIAL}

En materia de Seguridad Social existen disposiciones que en el futuro permitirán la creación de una jurisdicción contencioso - administrativa en materia de seguridad social, distinta de la jurisdicción contencioso administrativa general.

Con la jurisdicción contencioso administrativa se permite garantizar el control judicial sobre la actividad administrativa, pero como lo manifiesta Hernández ${ }^{8}$ (2006), la justicia administrativa pivota sobre un deficiente basamento legal y un desarrollo jurisprudencial.

De acuerdo con lo previsto en la Constitución de la República Bolivariana de Venezuela (artículo 259), los órganos de la jurisdicción contencioso - administrativa, son competentes para anular los actos administrativos generales o individuales contrarios a derecho, incluso por desviación de poder; condenar al pago de sumas de dinero y a la reparación de daños y perjuicios originados en responsabilidad de la

8 Hernández, J., "La pretensión procesal administrativa frente a las vías de hecho", en Derecho Contencioso Administrativo, Librerías Jurídicas Rincón. Barquisimeto 2001. El autor explana lo siguiente: "El desarrollo de la justicia administrativa en Venezuela ha mostrado una evidente dicotomía entre sus bases constitucionales y sus fundamentos legales. (...) Son esas, entonces, las dos peculiaridades sobre las que pivota la justicia administrativa en Venezuela: deficiente basamento legal (ante la ausencia de una ley de la jurisdicción contencioso - administrativa) y desarrollo principalmente jurisprudencial. Muchos han sido los avances que la jurisprudencia ha alcanzado, pero aun asi, todavía persisten ciertas áreas obscuras, en las cuales la defensa de los derechos e intereses de los particulares y el control de la Administración ha sido bastante parco".
Administración; conocer de reclamos por la prestación de servicios públicos y disponer lo necesario para el restablecimiento de las situaciones jurídicas subjetivas lesionadas por la actividad administrativa.

La jurisdicción contencioso administrativa de la Seguridad Social se encuentra contemplada en el artículo 141 de la Ley Orgánica del Sistema de Seguridad Social y se ratifica en la disposición transitoria décima quinta de la Ley del Régimen Prestacional de Empleo y en la disposición transitoria séptima de la Ley Orgánica de Prevención, Condiciones y Medio Ambiente del Trabajo, sin embargo este mandato constitucional y legal se encuentra en una evidente contradicción con el postulado contenido en el artículo 29 de la Ley Orgánica Procesal del Trabajo, que le otorga competencia a los Tribunales del Trabajo para conocer los asuntos de carácter contencioso que se susciten con ocasión de las relaciones laborales como hecho social, de las estipulaciones del contrato de trabajo y de la seguridad social.

La existencia de la jurisdicción contencioso administrativa permite garantizar el control efectivo de las actuaciones de la administración y a su vez se erige como garantía del acceso a la justicia y la tutela judicial efectiva. Duque ${ }^{9}$ (2001) en relación con el constitucionalismo del proceso contencioso administrativo ha indicado que el principio de tutela judicial efectiva permite extender el control jurisdiccional a los otros poderes, mediante el control contencioso administrativo.

Ahora bien, la competencia atribuida a la jurisdicción ordinaria laboral en materia contencioso - administrativa de la seguridad social, lejos de solucionar el problema del acceso a la justicia y la tutela judicial efectiva, pudiera generar un efecto perverso en el control judicial de las

9 Duque, R., "El constitucionalismo de un Estado de derechos y el proceso contencioso administrativo", en Estudios de Derecho Administrativo, Vol. I, Ediciones Tribunal Supremo de Justicia. Colección Libros Homenajes $\mathrm{N}^{\circ} 2$, Caracas 2001 . 
actividades administrativas del Servicio Público de Seguridad Social.

\section{CONCLUSIÓN}

El Sistema de Seguridad Social venezolano, diseñado bajo la forma de Servicio Público, se encuentra consagrado como norma programática en el artículo 86 constitucional; sin embargo, el texto constitucional de 1999 al concebir al Estado bajo los principios de un Estado Social de Derecho y de Justicia, implica en materia de Seguridad Social la interrelación de un conjunto de normas que articuladamente van a permitir el funcionamiento del Servicio Público de Seguridad Social.

En relación con la estructura organizativa, el Sistema de Seguridad Social no solamente se encuentra conformado por el órgano rector (Presidencia de la República), la Tesorería de la Seguridad y la Superintendencia de la Seguridad Social, debido a que existen adicionalmente los Sistemas Prestacionales y los Regimenes Prestacionales, que se encuentran a se vez integrados por un conjunto de órganos, entes, dependencias, organizaciones; así, como personas jurídicas de derecho público que coadyuvaran al desarrollo de las actividades administrativas en materia de seguridad social.

Uno de las ausencias que a juicio del investigador, repercuten significativamente en el optimo funcionamiento del Servicio Público de Seguridad Social, son las omisiones, imprecisiones y contradicciones normativas que presenta el articulado que regula el funcionamiento del Sistema de Seguridad Social, (actividades administrativas) trayendo como resultado, la preeminencia de la discrecionalidad por encima del principio de legalidad. Igualmente es significativo destacar la omisión del Estado en la conformación de un sistema contencioso administrativo que asegure a los usuarios una instancia judicial que permita el aseguramiento del acceso a la justicia y la tutela judicial efectiva. Pero no solamente es la omisión en la conformación del sistema contencioso administrativo, sino adi- cionalmente las omisiones en la aprobación de una Ley del sistema contencioso administrativo que consagre los procesos judiciales, simples, breves y expeditos, que permitan el disfrute del derecho humano a la seguridad social.

\section{BIBLIOGRAFÍA}

Constitución de la República Bolivariana de Venezuela, Gaceta Oficial 5.453, Marzo 24, 2000.

DÍAZ, L. ¿Acumulación de pobreza o reparto de riqueza?: El dilema de la seguridad social en Venezuela. Revista de Ciencias Sociales, mayo 2003, Vol IX, No. 2, p. 215-234. ISSN 1315-9518.

HERNÁNDEZ, J., "La pretensión procesal administrativa frente a las vías de hecho", en Derecho Contencioso Administrativo, Librerías Jurídicas Rincón. Barquisimeto 2001.

Ley de Servicios Sociales, Gaceta Oficial N. 38.270, Septiembre 12, 2005.

Ley del Régimen Prestacional de Empleo, Gaceta Oficial N. 38.281, Septiembre 27, 2005.

Ley del Régimen Prestacional de Vivienda y Hábitat, Gaceta Oficial N. 38.204, Junio 8, 2005.

Ley Orgánica de Procedimientos Administrativos, Gaceta Oficial N. 2828, Julio 1, 1981.

Ley Orgánica del Sistema de Seguridad Social, Gaceta Oficial N. 37.600, Diciembre 30, 2002

Ley Orgánica de Prevención, Condiciones y Medio Ambiente de Trabajo, Gaceta Oficial N. 38236, Julio 26, 2005.

Ley Orgánica del Trabajo. Gaceta Oficial N ${ }^{\circ}$ 5.152, Junio 19, 1997.

MÉNDEZ, A. "Estructura Organizativa y Funcional del Sistema de Seguridad Social", en Cuadernos de Postgrado N. 18, Comisión de Estudios de Postgrado, Universidad Central de Venezuela, Publicaciones UCV, Caracas 2004. 